\title{
Cloning and characterization of ribulose-1,5-bisphosphate carboxylase/oxygenase small subunit (RbcS) cDNA from green microalga Ankistrodesmus convolutus.
}

\begin{abstract}
An initial study on gene cloning and characterization of unicellular green microalga Ankistrodesmus convolutus was carried out to isolate and characterize the full-length cDNA of ribulose-1,5-bisphosphate carboxylase/oxygenase small subunit (RbcS) as a first step towards elucidating the structure of $\mathrm{A}$. convolutus $\mathrm{RbcS}$ gene. The full-length of $\mathrm{A}$. convolutus RbcS cDNA (AcRbcS) contained 28 bp of 5' untranslated region (UTR), 225 bp of $3^{\prime}$ non-coding region, and an open reading frame of 165 amino acids consisting of a chloroplast transit peptide with 24 amino acids and a mature protein of 141 amino acids. The amino acid sequence has high identity to those of other green algae RbcS genes. The AcRbcS contained a few conserved domains including protein kinase $\mathrm{C}$ phosphorylation site, tyrosine kinase phosphorylation site and $\mathrm{N}$-myristoylation sites. The AcRbcS was successfully expressed in Escherichia coli and a $\sim 21 \mathrm{kDa}$ of anticipated protein band was observed on SDS-PAGE. From the phylogenetic analysis of RbcS protein sequences, it was found that the $\mathrm{RbcS}$ of $\mathrm{A}$. convolutus has closer genetic relationship with green microalgae species compared to those of green seaweed and green macroalgae species. Southern hybridization analysis revealed that the AcRbcS is a member of a small multigene family comprising of two to six members in A. convolutus genome. Under different illumination conditions, RTPCR analysis showed that AcRbcS transcription was reduced in the dark, and drastically recovered in the light condition. Results presented in this paper established a good foundation for further study on the photosynthetic process of A. convolutus and other green algae species where little information is known on Rubisco small subunit.
\end{abstract}

Keyword: Green microalgae; Ankistrodesmus convolutus; cDNA library construction; Expressed sequence tags. 